\title{
Erythema Multiforme Secondary to HSV Labialis Precipitating Sickle Cell Pain Crisis
}

Lorenzo Di Francesco, MD

Emory University School of Medicine

A 28-year-old man with sickle cell anemia was admitted with generalized pain. He noted an upper lip lesion 2 weeks prior to admission. He subsequently developed generalized pain in his legs, chest, and back typical of his pain crises. At admission he noted subjective fevers without chills for a week. Vital signs revealed a blood pressure of $135 / 80$, a pulse of 81 , a respiratory rate of 16 , and an initial temperature of $37.7^{\circ} \mathrm{C}$. On examination he had scleral icterus and a large upper lip ulcer (Fig. 1). His hospital course was complicated by persistent fevers, a hepatic sequestration crisis, persistent hemolytic anemia requiring blood transfusion, and ultimately the identification of iris-shaped targetoid lesions on the palms (Fig. 2).These lesions were believed to be consistent with erythema multiforme (EM) secondary to his recent HSV labialis, confirmed by a herpes culture. The patient recovered uneventfully after a 10-day hospitalization. Erythema multiforme is an acute, self-limited, but sometimes recurrent dermatologic condition considered to be a distinct hypersensitivity reaction. ${ }^{1}$ It is associated with certain infections such as herpes simplex 1 and 2, Mycoplasma pneumoniae and fungal infections, and a number of medications in the

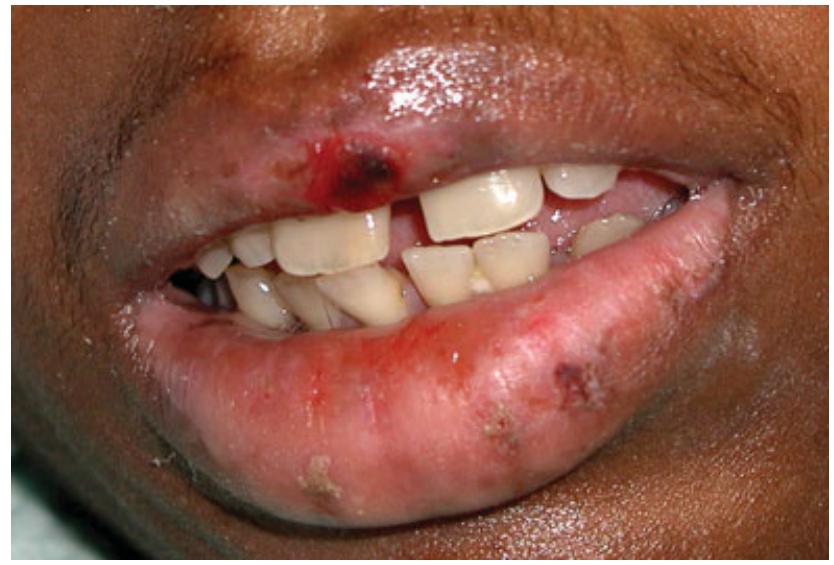

FIGURE 1. HSV labialis. classes barbiturates, nonsteroidal anti-inflammatory drugs, penicillins, hydantoins, phenothiazines, and sulfonamides. ${ }^{2}$ EM is diagnosed clinically by the characteristic rash on the hands and feet, with some cases involving the oral cavity. Treatment is typically focused on resolving the underlying infection or removing the offending drug. Dermatologic manifestations usually improve over 3-5 weeks without residual sequelae.

\section{REFERENCES}

1. Aurelian L, Kokuba H, Burnett JW. Understanding the pathogenesis of HSV-associated erythema multiforme. Dermatology. 1998;197:219-222.

2. Lamoreux MR, Sternbach MR, Hsu WT. Erythema multiforme. Am Fam Physician. 2006;74:1883-1888.

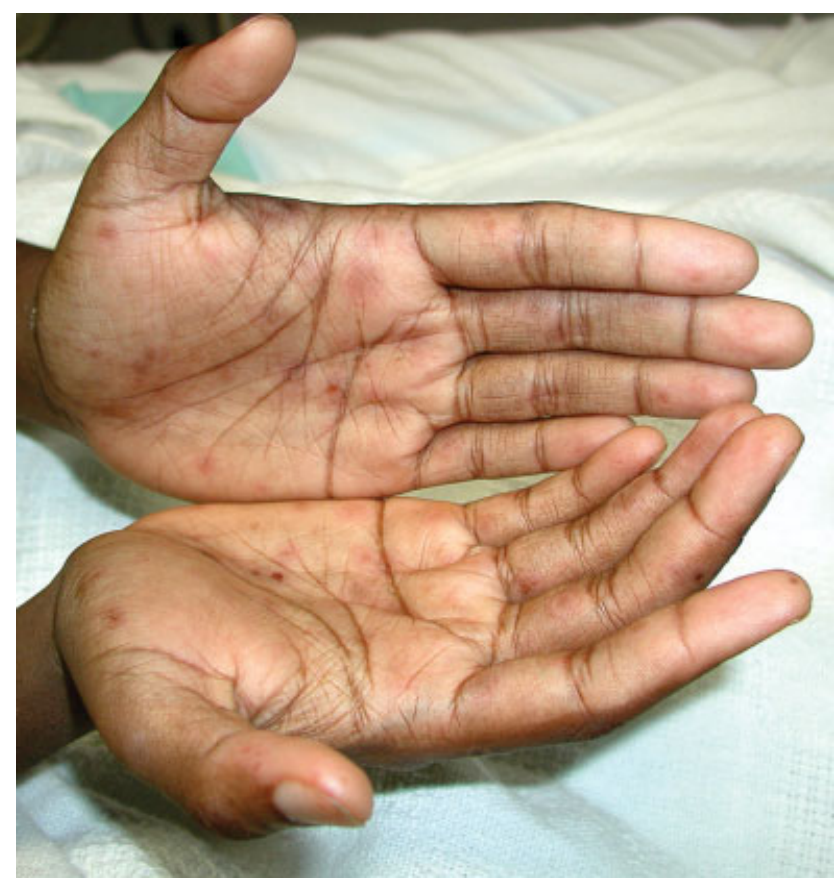

FIGURE 2. Erythema multiforme. 